\title{
FUNDAMENTAL LIMITS ON PHASE AND FREQUENCY TRACKING AND ESTIMATION IN DRIFTING OSCILLATORS
}

\author{
D. R. Brown III* R. Mudumbai and S. Dasgupta ${ }^{\dagger}$
}

\begin{abstract}
Distributed beamforming requires phase and frequency synchronization. As oscillators drift, through Brownian motion induced phase noise, their instantaneous phases must be tracked and compensated. Several papers and IEEE 1588 have proposed Kalman Filter (KF) based tracking algorithms using the unwrapped phase measurements. This paper quantifies the effect of Brownian Motion induced drift at two levels. First we derive Cramer-Rao Lower Bounds (CRLB) manifest in one shot estimation of frequency and phase from unwrapped phase observations, and reveal fundamental and illuminating differences with the existing frequency and phase estimation CRLBs in the literature derived in the absence of Brownian motion. Second, we consider a KF that tracks the instantaneous phase in intervals where there is no beamforming, and is switched off during beamforming. Bounds are derived relating the error growth as a function of the underlying duty cycle.
\end{abstract}

Index Terms - Oscillator Drift, Phase Noise, Brownian Motion, Kalman Filter, Cramer Rao Lower Bound

\section{INTRODUCTION}

Distributed beamforming requires phase and frequency synchronization. Even without mobility, oscillators undergo drift that must be tracked and compensated. The process characterizing this drift is Brownian motion, typically in both phase and frequency [1]-[3]. This causes the variance of the phase offset with respect to an ideal reference to grow with time.

The stochastic nature of independent local oscillators implies that it is not just enough to estimate the channels from the transmit nodes (which include the effect of clock offsets) and expect them to hold for long, even without motion. In techniques that require close synchronization of transmit nodes, uncorrected Brownian motion oscillator drift also leads to desynchronization. Oscillator offsets must be tracked and appropriately compensated, else channel estimates become stale, transmit nodes become unsynchronized, and the gains of distributed transmission are lost.

*ECE Dept Worcester Polytechnic Inst., Worcester, MA 01609, USA, drb@wpi.edu

${ }^{\dagger}$ ECE Dept Univ. of Iowa, Iowa City IA 52242, USA, [rmudumbai,dasgupta]@engineering.uiowa.edu. Supported by grants from NSF ECS0622017, CCF-07290 and CCF-0830747, and the Carver Charitable Trust.
Consider a WiFi distributed beamforming system at a carrier frequency of $2.4 \mathrm{GHz}$ with ten fixed transmit nodes and one fixed receive node. Suppose at time $t=0$, the transmit nodes are perfectly synchronized and have perfect channel state knowledge. The received power at $t=0$ is $10 \log _{10}\left(10^{2}\right)=20 \mathrm{~dB}$. Even though there is no motion in the system, the oscillators will begin to drift for time $t>0$. Oscillators used in WiFi transceivers typically drift with a standard deviation of of 2.5 nanoseconds per second [5]. At $2.4 \mathrm{GHz}$, this corresponds to a phase standard deviation of 108 degrees at time $t=0.050 \mathrm{~s}$, which corresponds to an average received power of appoximately $11 \mathrm{~dB}(1 \mathrm{~dB}$ better than incoherent transmission). In other words, even though the standard deviation of the clocks offsets was only 125 picoseconds at $t=0.050 \mathrm{~s}$, this is manifested as a large phase standard deviation, and consequently poor beamforming, at typical radio frequencies.

Thus, in order to maintain good performance in distributed transmit beamforming systems, it is important to understand how frequently one must resynchronize. Recent papers including [1,2] and IEEE 1588, [7] have adopted a two-state Kalman filtering approach, in which they assume that the unwrapped phase is available, and track phase and frequency using these unwrapped phase measurements. The efficacy of such a Kalman filter based approach to distributed transmit beamforming has also recently been demonstrated in [6].

This paper is focused on acquiring a fundamental understanding of the effect of Brownian motion driven phase drift at two levels. In the first we derive Cramer-Rao Lower Bounds (CRLB) manifest in one shot estimation of frequency and phase from instantaneous observations of the unwrapped phase, and reveal fundamental and illuminating differences with the existing frequency and phase estimation CRLBs in the literature, e.g. [4], derived assuming the absence of phase drift. At the second level we consider the use of a Kalman filter that tracks the instantaneous unwrapped phase of a single oscillator, in intervals where there is no beamforming, and is inevitably switched off during beamforming. Bounds are derived relating the error growth as a function of the underlying duty cycle.

\section{MODELS}

Our focus here is to study the effect of Brownian motion induced phase noise. Over short observation intervals, the ef- 
fect of Brownian motion frequency noise can be assumed to be negligible [3]. Thus, the basic model we consider is one in which the unwrapped phase obeys

$$
y\left(n T_{s}\right)=\omega_{c} n T_{s}+\theta+\sum_{i=-p}^{n-1} w\left(i T_{s}\right)+v\left(n T_{s}\right)
$$

where $\omega_{c}$ is the nominal oscillator frequency, $\theta$ a nominal phase, $T_{s}$ is the sampling interval, $w\left(i T_{s}\right) \sim N\left(0, \omega_{c}^{2} T_{s} q=\right.$ $\left.\sigma_{u}^{2}\right)$ is a white sequence that models the Brownian motion, and $v\left(n T_{s}\right) \sim N\left(0, \sigma_{v}^{2}\right)$ is also a white sequence, modeling the effect of $\eta$ and phase unwrapping errors. In the sequel define, $y[n]=y\left(n T_{s}\right), w[n]=w\left(n T_{s}\right)$ and $v[n]=v\left(n T_{s}\right)$. The fact that the summation in (1) starts at $i=-p$, reflects the fact that the first observation made is $p$ samples after the phase noise takes effect.

A state variable respresention for (1) is:

$$
x[k+1]=F x[k]+G w[k] \quad y[k]=H x[k]+v[k],
$$

where

$$
x[k]=\left[\begin{array}{c}
\theta[k] \\
\omega_{c}
\end{array}\right] \quad F=\left[\begin{array}{cc}
1 & T_{s} \\
0 & 1
\end{array}\right] \quad G=\left[\begin{array}{l}
1 \\
0
\end{array}\right] \quad H=\left[\begin{array}{ll}
1 & 0
\end{array}\right] .
$$

\section{CRAMER RAO LOWER BOUND}

The past literature on CRLBs for frequency and phase estimation, e.g. [4], involves observations of complex exponentials in white noise, without oscillator drift. Specifically, the focus is to study the estimation of $\omega_{c}$ and $\theta$ from

$$
z(t)=r e^{\left(j \omega_{c} t+\theta\right)}+\eta(t)
$$

where $\eta(t)$ is complex Gaussian noise. Analysis of the effect of the statistics of $\eta$, the sampling rate and the number of samples is given. As our goal is to understand the effect of Brownian motion, rather than studying the effect of $\eta$, we derive the CRLBs by neglecting $v\left(n T_{s}\right)$ in (1) or equivalently $v[n]$ in (2). We work instead with

$$
y[n]=\omega_{c} n T_{s}+\theta+\sum_{i=-p}^{n-1} w[i] .
$$

Our goal is to obtain the CRLB in estimating $\omega_{c}$ and $\theta$ from the $N$-observation vector $Y_{N}=[y[0], \cdots, y[N-1]]^{T}$. To this end observe that with

$$
W_{N}=\left[\sum_{i=-p}^{0} w[i], \cdots, \sum_{i=-p}^{N-1} w[i]\right]^{T}
$$

there holds: $Y_{N}=\omega_{c} T_{s} s_{N}+\theta u_{N}+W_{N}$, with the $N$-vectors $s_{N}=[0,1,2, \cdots, N-1]^{T}$ and $u_{N}=[1,1, \cdots, 1]^{T}$. Observe, $W_{N} \sim N\left(0, \Sigma_{N}\right)$, where the $\{i j\}$-th element, $i \leq j$, of the symmetric matrix $\Sigma_{N}=E\left[W_{N} W_{N}^{T}\right]$ obeys:

$$
\Sigma_{N}(i, j)=(i+p) \omega_{c}^{2} T_{s} q
$$

For example with $N=4$

$$
\Sigma_{N}=\omega_{c}^{2} T_{s} q\left[\begin{array}{cccc}
p+1 & p+1 & p+1 & p+1 \\
p+1 & p+2 & p+2 & p+2 \\
p+1 & p+2 & p+3 & p+3 \\
p+1 & p+2 & p+3 & p+4
\end{array}\right] .
$$

Thus the conditional density function $f_{Y_{N} \mid \Omega_{c}, \Theta}\left(Y_{N} \mid \omega_{c}, \theta\right)$ is $N\left(\omega_{c} T_{s} s_{N}+\theta u_{N}, \Sigma_{N}\right)$. Then, [8], the Fisher Information Matrix (FIM) is given by:

$$
F_{N}=\left[\begin{array}{cc}
T_{s}^{2} s_{N}^{T} \Sigma_{N}^{-1} s_{N} & -T_{s} s_{N}^{T} \Sigma_{N}^{-1} u_{N} \\
-T_{s} s_{N}^{T} \Sigma_{N}^{-1} u_{N} & u_{N}^{T} \Sigma_{N}^{-1} u_{N}
\end{array}\right]
$$

where, with $z_{1}=\omega_{c}$ and $z_{2}=\theta$, the $(i, j)$-th element of $F_{N}$ represents $-E\left\{\partial^{2} \log \left[f_{Y_{N} \mid \Omega_{c}, \Theta}\left(Y_{N} \mid \omega_{c}, \theta\right)\right] / \partial z_{i} \partial z_{j}\right\}$. Direct verification shows that:

$$
\Sigma_{N}[1,0, \cdots, 0]^{T}=(p+1) \omega_{c}^{2} T_{s} q u_{N}
$$

Likewise:

$$
\Sigma_{N}[-1,0, \cdots, 0,1]^{T}=\omega_{c}^{2} T_{s} q s_{N}
$$

Subsituting into (9) we get,

$$
F_{N}=\frac{1}{\omega_{c}^{2} q} \operatorname{diag}\left\{(N-1) T_{s}, \frac{1}{(p+1) T_{s}}\right\} .
$$

The CRLB matrix is then:

$$
\omega_{c}^{2} q \operatorname{diag}\left\{\frac{1}{(N-1) T_{s}},(p+1) T_{s}\right\}
$$

where the first and second diagonals are the CRLB for frequency and phase estimation, respectively. By contrast the CRLB matrix for estimating $\omega_{c}$ and $\theta$ from (4) is:

$$
\frac{\sigma^{2}}{r^{2}}\left[\begin{array}{cc}
\frac{1}{T_{s}^{2} N\left(Q-P^{2}\right)} & \frac{-(p+P)}{T_{s} N\left(Q-P^{2}\right)} \\
\frac{-(p+P)}{T_{s} N\left(Q-P^{2}\right)} & \frac{p^{2}+2 p P+Q}{N\left(Q-P^{2}\right)}
\end{array}\right]
$$

where $\sigma^{2}$ is the variance of the uncorrelated real and imaginary components of $\eta, P:=(N-1) / 2, Q:=(N-1)(2 N-$ $1) / 6$. The most striking thing about the comparison between (11) and (12) is that in the estimation of frequency, despite the fact that the computation of (11) neglects phase unwrapping errors, the improvement with the number of observations is only linear, as opposed to quadratic in (12), reflecting the influence of Brownian motion. Further, while the CRLB for $\theta$ is unaffected by the number of observations in the Brownian motion case, it grows linearly with $T_{s}$. By contrast, without Brownian motion, $N$ does affect the phase estimation CRLB, but $T_{s}$ does not.

\section{KALMAN TRACKING}

In the rest of this paper we consider state tracking performance within a two-stage format. Specifically we will assume 
that there are two interlaced epochs that are repeated periodically. In the first, the training or tracking epoch the Kalman filter (KF) runs using the unwrapped phase measurements to track the actual state in (2). In the second the idling or beamforming epoch, it is idle. For positive integers $L>N$ and all natural numbers $M$, the tracking epoch comprises samples $\{M L, M L+1, \cdots, M L+N-1\}$, and the idling epoch comprises $\{M L+N, M L+N+1, \cdots, M L+L-1\}$. The duty cycle involved is $D=(L-N) / L$. Both, $x[k]$ and $y[k]$ are now offsets from some initial estimates.

Define $\hat{x}[k+1 \mid k]$ to be the state prediction estimate obtained at time $k+1$ on the basis of observations $y[0], \cdots, y[k]$ and $\hat{x}[k \mid k]$ to be the state prediction estimate obtained at time $k$ on the basis of observations $y[0], \cdots, y[k]$. Observe, the idling epoch precludes the use of steady state Kalman gain. Rather in the tracking epoch we use the standard KF equations as used in the controls literature ${ }^{1}$, [9]. With $\sigma_{u}^{2}=\omega_{c}^{2} T_{s} q$, stated purely as a recursion in terms of $\hat{x}[k+1 \mid k]$, this becomes:

$$
\begin{gathered}
\hat{x}[k+1 \mid k]=\left(F-K[k] H^{T}\right) \hat{x}[k \mid k-1]+K[k] y[k] . \\
K[k]=F \Sigma[k \mid k-1] H^{T}\left(H \Sigma[k \mid k-1] H^{T}+\sigma_{v}^{2}\right)^{-1} \\
\Sigma[k+1 \mid k]=F\left[\Sigma[k \mid k-1]-\Sigma[k \mid k-1]\left(H \Sigma[k \mid k-1] H^{T}\right.\right. \\
\left.\left.+\sigma_{v}^{2}\right)^{-1} H \Sigma[k \mid k-1]\right] F^{T}+\sigma_{u}^{2} G G^{T}
\end{gathered}
$$

Specifically, $\Sigma[k+1 \mid k]$ represents the error covariance matrix at time $k+1$, based on the observations $y[0], \cdots, y[k]$. In the sequel we quantify performance on the basis of $\Sigma[k+1 \mid k]$, as the obviously defined $\Sigma[k \mid k]$ is readily obtained from it. In the idle epoch, in the absence of training, the correct place holder for the state estimate used to initialize at the next tracking epoch is:

$$
\hat{x}[k+1 \mid k]=F \hat{x}[k \mid k-1] .
$$

Figure 1 exemplifies the performance of the Kalman filter versus the one-shot maximum likelihood estimator [10] with a phase process noise parameter selected to yield a standard deviation of 108 degrees of offset after 50ms (as is typical for WiFi transceivers as discussed in Section 1$) ; \sigma_{v}^{2}=0.6169$. In this example, each tracking epoch is $0.5 \mathrm{~ms}$, followed by an idle epoch of $4.5 \mathrm{~ms}$ with $T_{s}=10 \mu \mathrm{s}$. After the first tracking interval, the KF and the one-shot estimators perform similarly during the idle epoch. The advantage of the KF becomes evident after subsequent observations where the $\mathrm{KF}$ incorporates the prior knowledge with the new observations. In steady state, the prediction error of the $\mathrm{KF}$ at the end of the idle epoch is less than 30 degrees RMS, which provides a beamforming gain less than $1.5 \mathrm{~dB}$ of the ideal with 10 transmit nodes [6]. The MLE has an RMS phase error in excess of 200 degrees at the end of each idle epoch, corresponding

\footnotetext{
${ }^{1}$ There is a slight difference in terminology between the controls and estimation literature, though the end products are equivalent
}

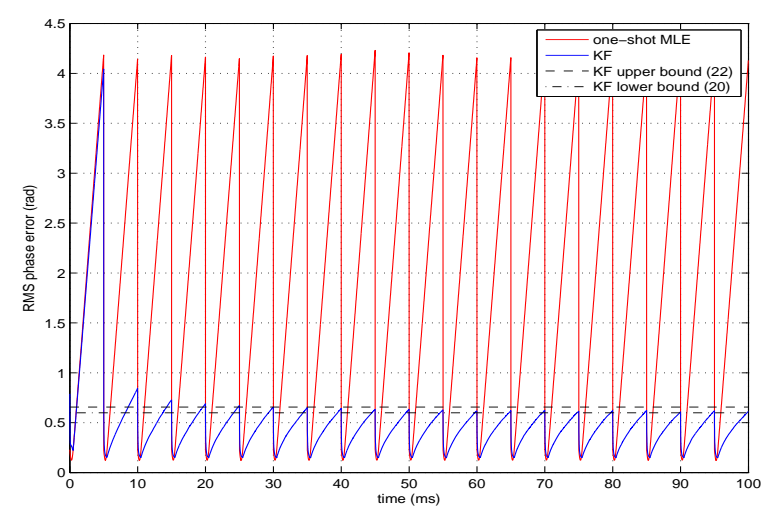

Fig. 1. One shot ML tracking vs interlaced KF tracking with steady state error bounds for KF tracking.

to incoherent combining. The black lines will be explained in the next section. The results are averaged over $10^{4}$ iterations.

\section{KALMAN FILTER PERFORMANCE}

During the training epoch, i.e. for $k \in\{M L, M L+$ $1, \cdots, M L+N-1\}$, (13) holds, while during the idle epoch, i.e. for $k \in\{M L+N, M L+N+1, \cdots, M L+L-1\}$, there obtains

$$
\Sigma[k+1 \mid k]=F \Sigma[k \mid k-1] F^{\prime}+\sigma_{u}^{2} G G^{\prime} .
$$

We begin by making some important remarks. Most proofs of KF convergence, even with no idling, assume that $[F, G]$ is stabilizable. In this case $[F, G]$ is not stabilizable, let alone completely controllable. However, the fact that the uncontrolable mode is simple and at 1, implies, [12], that the complete observability of $[F, H]$ ensures that had we only trained, i.e. only (13) held, $\Sigma[k \mid k-1]$ would still converge to a steady state value, that obeys the standard algebraic Riccati equation. What the lack of stabilizability does is to guarantee that the resulting steady state Kalman gain $K$ is such that $F-K H$ has one eigenvalue at 1 .

In this case one has to contend with the idle epoch as well. Such a setting has not been analyzed in the case where $[F, G]$ is not stabilizable. The setting where $[F, G]$ is stabilizable has been considered in the sensor scheduling literature, e.g. [11], but only for $N=1$. Here we consider a general $N$ and show that at steady state this results in a periodic solution. In fact we have the following Theorem.

Theorem 5.1 For every pair of integers $L>N>1$, scalars $\sigma_{u}^{2}>0$ and $\sigma_{v}^{2} \geq 0$, there exists a unique $a>0$ such that the periodic scalar sequence $a[k]$ defined by the following is unique and well defined: (a) For all natural numbers $M$, $a[M L]=a$. (b) For all $k \in\{M L, M L+1, \cdots M L+N-1\}$

$$
a[k+1]=a[k]-\frac{a^{2}[k]}{\sigma_{v}^{2}+a[k]}+\sigma_{u}^{2}
$$


(c) There holds:

$$
a[M L+N]+(L-N) \sigma_{u}^{2}=a .
$$

Further, consider the $2 \times 2$ symmetric matrix sequence $\Sigma[k \mid k-1]$, that under (3), obeys (13) for all $k \in\{M L, M L+$ $1, \cdots M L+N-1\}$, and (15) for $k \in\{M L+N, M L+N+$ $1, \cdots, M L+L-1\}$, with symmetric $\Sigma[0 \mid-1] \geq 0$. Then:

$$
\lim _{k \rightarrow \infty}\|\Sigma[k+1 \mid k]-\operatorname{diag}\{a[k], 0\}\|=0 .
$$

Evidently, the frequency estimation error goes to zero. This is unsurprising as the second state in (2) is constant. The phase tracking error variance at steady state is periodic. The largest value, at the end of the idle period is given by $a$. We now provide bounds on $a$. To this end observe that (16) is a first order Riccati equation with steady state solution:

$$
a^{*}=\left(\sigma_{u}^{2}+\sqrt{\sigma_{u}^{4}+4 \sigma_{v}^{2} \sigma_{u}^{2}}\right) / 2 .
$$

From (17), $a[L M+N]<a$. Then as $a[k]$ in (16) is monotonic the solution to (16) is nonincreasing in the interval $[L M, L M+N-1]$. Thus $a[L M+N] \geq a^{*}$, and

$$
a \geq(L-N) \sigma_{u}^{2}+\left(\sigma_{u}^{2}+\sqrt{\sigma_{u}^{4}+4 \sigma_{v}^{2} \sigma_{u}^{2}}\right) / 2 .
$$

Also observe that from the nondecreasing and nonegative nature of the sequence in (16), in the tracking epoch $a[k] \geq a^{*}$. Thus with

$$
\begin{gathered}
0 \leq \lambda=\frac{\sigma_{v}^{2}}{\sigma_{v}^{2}+a^{*}}<1 . \\
a[k+1]=\frac{\sigma_{v}^{2}}{\sigma_{v}^{2}+a[k]} a[k]+\sigma_{u}^{2} \leq \lambda a[k]+\sigma_{u}^{2}
\end{gathered}
$$

Then using the fact that $a[L M]=a$, a repeated recursion shows that

$$
a[L M+N] \leq \lambda^{N} a+\sigma_{u}^{2} \frac{1-\lambda^{N}}{1-\lambda} .
$$

Consequently using (17), we obtain:

$$
a \leq \sigma_{u}^{2}\left(\frac{L-N}{1-\lambda^{N}}+\frac{1}{1-\lambda}\right) .
$$

Observe if $\sigma_{v}^{2}=0$, then the two bounds coincide, i.e. under high SNR the bounds are arbitrarily tight. These also quantify the effect of duty cycle $D, L$ and $N$. In Figure 1 the steady state error evidently matches the lower bound, and the two bounds are quite close.

\section{CONCLUSION}

Motivated by stringent carrier synchronization requirements for beamforming, we have studied the effect of Brownian motion induced phase noise. Influenced by IEEE 1588's adoption of a Kalman Filter based tracking of carrier noise using unwrapped phase measurements, we have studied two fundamental issues. We have derived the CRLB for estimating the phase and frequency from the unwrapped phase and have delineated notable differences from existing CRLBs for phase and frequency estimation in the absence of Brownian motion. We have studied the performance of a two stage Kalman Filter, interlacing periods of idling and tracking. We have shown that the steady prediction error covariance matrix is periodic, and have provided bounds on its value.

\section{REFERENCES}

[1] C. Zucca and P. Tavella, "The clock model and its relationship with the allan and related variances," $U l$ trasonics, Ferroelectrics and Frequency Control, IEEE Transactions on, vol. 52, pp. 289 -296, feb. 2005.

[2] L. Galleani, "A tutorial on the two-state model of the atomic clock noise," Metrologia, 2008.

[3] J. A. McNeill and D. Ricketts, The Designer's Guide to Jitter in Ring Oscillators, Springer, 2009.

[4] D. Rife and R. Boorstyn, "Single-tone parameter estimation from discrete-time observations," IEEE Transactions on Information Theory, pp. 591-598, 1974.

[5] "IEEE Standard 802.1AS-2011 for local and metropolitan area networks - timing and synchronization for time-sensitive applications in bridged local area networks," 2011.

[6] D.R. Brown, P. Bidigare, and U. Madhow, "ReceiverCoordinated Distributed Transmit Beamforming with Kinematic Tracking", ICASSP 2012, to appear.

[7] IEEE Standard for a Precision Clock Synchronization Protocol for Networked Measurement and Control Systems, IEEE 2008.

[8] H. Van Trees, Detection, Estimation, and Modulation Theory, Part I. New York: John Wiley and Sons, 1968.

[9] B. D. O. Anderson and J. B. Moore, Optimal Filtering. Prentice Hall, 1979.

[10] S. A. Tretter, "Estimating the frequency of a noisy sinusoid by linear regression", IEEE Transactions on Information Theory, 1985

[11] L. Shi, K. J. Henriksson and L. Qiu, "Time and Eventbased Sensor Scheduling for Networks with Limited Communication Resources", in Preprints of IFAC World Congress, Milano, Italy 2011.

[12] B. D. O. Anderson, "Stability properties of KalmanBucy filters", Journal of Franklin Institute, 1971. 